\title{
MACteaching: Utilizando um Jogo para Apoio ao Ensino do Método de Avaliação de Comunicabilidade
}

\author{
Ruddá Silva Beltrão Brito ${ }^{1}$, Martha Fernandes ${ }^{2}$, Wagner Queiróz ${ }^{1}$, Bruno P. de \\ Souza $^{1}$, Bruno A. Bonifácio ${ }^{1}$, Priscila Fernandes ${ }^{1,3}$ \\ ${ }^{1}$ Instituto de Ciências Exatas e Tecnologia (ICET) - Universidade Federal do Amazonas \\ (UFAM) - Caixa Postal 15.064 - 91.501-970 - Itacoatiara - AM - Brazil \\ ${ }^{2}$ Instituto Federal do Amazonas (IFAM) - Caixa Postal 100.665 - Manaus - AM-Brazil \\ ${ }^{3}$ Grupo de Usabilidade e Engenharia de Software (USES) - Universidade Federal do \\ Amazonas (UFAM)

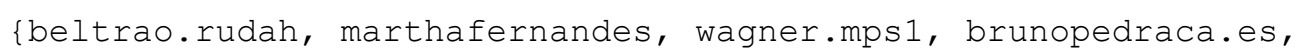

\begin{abstract}
The use of software technologies within educational environment has created new teaching practices to facilitate the teaching and learning process. This scenario contributed to the growth in the number of increasingly interactive mobile applications. New applications that meet the expectations of users brings new challenges to the courses of Information Technology (IT). In this context, the Human-Computer Interaction area (HCI) brings an innovative vision of the application creation process. However teach HCI is no easy task. This paper presents the MACteaching, a game that aims to complement the teaching of communicability evaluation method, a HCI method, in a playful manner. Besides presenting the game, this paper describes its development process and its empirical evaluation, improvements to assist HCI courses.
\end{abstract}

Resumo. A utilização de tecnologias de software dentro do ambiente educacional tem criado novas práticas pedagógicas para facilitar o processo de ensino e aprendizagem. Esse cenário contribuiu para o crescimento do número de aplicativos móveis desenvolvidos para apoiar este processo. Criar novas aplicações, que atendam as expectativas dos usuários traz novos desafios para os cursos de Tecnologia da Informação (TI). Nesse contexto, a área Interação Humano-Computador (IHC) traz uma visão inovadora sobre o processo de criação de aplicativos. Todavia ensinar IHC não é tarefa fácil. $O$ presente trabalho apresenta o MACteaching, um jogo que visa auxiliar o ensino do Método de Avaliação de Comunicabilidade, um método de IHC, de forma lúdica. Além de apresentar o jogo, este artigo descreve o processo de desenvolvimento e a avaliação experimental de ensino nas disciplinas de IHC.

\section{Introdução}

Dentro do campo educacional, o crescente uso de tecnologia de apoio ao ensino tem criado novas práticas pedagógicas para facilitar o processo de ensino e aprendizagem [Meireles e Bonifácio 2015]. Essa transformação impulsionou o surgimento de novas formas de interação e aprendizado, principalmente com o surgimento dos dispositivos e aplicações móveis. Devido essa diversidade de tecnologias surge a necessidade de criação de aplicações cada vez mais interativas, para atender a expectativa dos usuários [Coutinho 
V Congresso Brasileiro de Informática na Educação (CBIE 2016)

Anais do XXVII Simpósio Brasileiro de Informática na Educação (SBIE 2016)

et al. 2015]. Porém, saber como criar novas aplicações, que atendam as expectativas dos usuários, exige conhecimento, técnicas, ferramentas e métodos que englobam tanto a Engenharia de Software como a área Interação Humano-Computador (IHC). A área de IHC fornece uma visão sobre o processo de desenvolvimento de sistemas interativos que foca na qualidade de interação usuário-sistema, considerando fatores relacionados aos usuários e como atender de maneira satisfatória suas necessidades e expectativas [Sommariva et al. 2011]. Assim, a área de IHC tem evoluído para propiciar aos desenvolvedores e estudantes um corpo de conhecimento necessário para identificação de requisitos para construção de interfaces [Bim et al. 2012]. Por essa razão, o ensino de IHC tem sido cada vez mais difundido em cursos de graduação e pós-graduação na área de Tecnologia da Informação (TI), propiciando a formação de profissionais mais qualificados, e consequentemente, sistemas computacionais de melhor qualidade.

Para lidar com esses desafios, os centros de ensino têm se esforçado para criar um ambiente motivador, no qual seja possível ensinar aos estudantes e profissionais os fundamentos de IHC, por meio de materiais de ensino, exemplos e dinâmicas para aplicar durante as aulas. Assim, o uso de tecnologias móveis, como recurso complementar, no processo de ensino e aprendizagem pode auxiliar na criação de atividades pedagógicas mais colaborativas e inovadoras. Nesse contexto, jogos educativos podem auxiliar no desenvolvimento de habilidades cognitivas, para resolução de problemas, tomada de decisão, reconhecimento de padrões, processamento de informações, criatividade e pensamento crítico de forma lúdica. E com isso potencializar o aprendizado, possibilitar a fixação do conteúdo de forma mais eficaz e incentivar o aprendizado por meio de desafios.

Esse cenário motivou a elaboração do jogo para dispositivos móveis, chamado MACteaching. Essa solução utiliza uma abordagem de interação que visa melhorar a experiência de alunos no ensino de IHC, especificamente no ensino de um método fundamentado na Engenharia Semiótica, o Método de Avaliação de Comunicabilidade (MAC). A escolha do MAC é justificada devido à dificuldade que normalmente os alunos apresentam no ensino de etapas do método, o que constitui grande oportunidade de pesquisa. O MACteaching utiliza simulações para reforçar os conceitos do MAC.

Os objetivos desse artigo consistem em: 1) apresentar as características que tornam o jogo uma solução que pode contribuir para o processo de ensino e aprendizagem para a área de IHC e Engenharia de Software; 2) relatar o estudo realizado para avaliar o jogo em ambiente real; além de 3) identificar melhorias que serviram como base para aprimoramento da experiência de uso do jogo.

O artigo está organizado da seguinte forma: A Seção 2 descreve os conceitos e trabalhos relacionados a Engenharia Semiótica, o MAC e a jogos para ensino de IHC. A Seção 3 apresenta o jogo MACteaching. A Seção 4 apresenta a metodologia utilizada para realizar o estudo, a condução da avaliação realizada, e também discute os resultados obtidos. A 5 lições aprendidas e melhorias realizadas no jogo com base nos resultados. Por fim, a Seção 6 lista as conclusões e trabalhos futuros.

\section{Conceitos e Trabalhos Relacionados}

O aparecimento constante de novas tecnologias tem alterado a forma como os usuários interagem com os sistemas interativos; e consequentemente como as tecnologias de software são desenvolvidas. Todavia, desenvolver novos produtos, avaliá-los, e saber 
quando identificar melhorias são desafios para área de IHC. Nesse contexto, vários métodos para avaliar a qualidade de sistemas têm sido propostos. Dentre estes, estão os fundamentados na Engenharia Semiótica [Prates e Barbosa 2007]. A Engenharia Semiótica é uma teoria de IHC que caracteriza a interação usuário-sistema como um tipo específico de comunicação, categorizando aplicações computacionais como artefatos de metacomunicação [Barbosa e Silva 2010]. A metamensagem ocorre na comunicação existente entre usuário e designer, por meio da interface [Salgado et al. 2006]. O Método de Inspeção Semiótica e o Método de Avaliação de Comunicabilidade (MAC) são exemplos de métodos de avaliação fundamentados na Engenharia Semiótica.

\subsection{Método de Avaliação de Comunicabilidade (MAC)}

Devido sua relevância, o MAC tem sido amplamente adotado [de Souza 2005]. Este método tem como objetivo avaliar a qualidade da metacomunicação entre o designer e o usuário. De acordo com Barbosa e Silva (2010), o método possui cinco atividades: (1) preparação, onde devem ser realizados a definição das tarefas a serem executadas pelos participantes, definição do perfil e seleção os participantes; (2) coleta de dados, onde os usuários são observados durante a interação com o sistema interativo que está sendo avaliado e suas sessões são registradas; (3) interpretação, onde são atribuídas etiquetas, previamente definidas no MAC, para as rupturas extraídas dos vídeos da interação; (4) consolidação dos resultados, onde é realizada interpretação das etiquetagens e elaboração do perfil semiótico; e (5) relato dos resultados. O MAC possui um total de treze etiquetas as quais possuem contextos específicos. No processo de ensino, os estudantes comumente encontram dificuldade para utilização do método, como dificuldade na assimilação e diferenciação das etiquetas.

Apesar das iniciativas para o desenvolvimento de jogos para o ensino de IHC, a literatura não apresenta trabalhos específicos para ensino de conceitos de Engenharia Semiótica, especificamente do MAC. Por essa razão, optou-se por desenvolver um jogo que auxiliasse no ensino deste método.

\section{Caracterização do Jogo MACteaching}

O jogo educativo MACteaching tem como proposta facilitar o processo de ensino e aprendizagem do MAC. O MACteaching utiliza como abordagem interativa de aprendizagem relacional, por meio de associação por analogia. O propósito do jogo é ser uma ferramenta complementar ao ensino do método, focando em trabalhar os conceitos de forma lúdica. O jogo caracteriza-se como um simulador prático dos conceitos do MAC, pois apresenta cenários descrevendo casos de uso que são passíveis de acontecer durante a observação do participante, na aplicação do método. Além de prática, com exercícios que favorecem o ensino das etapas do método, apresentando de maneira rápida, novas problemáticas, para que o jogador possa se familiarizar rapidamente com as etiquetas e os conceitos da área. O jogo foi desenvolvido para plataforma Android.

A plataforma de desenvolvimento utilizada foi o Android Studio ${ }^{1}$, para criação e edição de imagens foi usado o Photoshop $\mathrm{CS}^{2}$. A interface do jogo MACteaching, apresentado na Figura 1, foi organizada da seguinte maneira: ao iniciar o jogo, o aluno é inserido na história do jogo, onde são apresentados os conceitos da área por meio de

${ }^{1}$ google.android.com

${ }^{2}$ photoshop.org 
V Congresso Brasileiro de Informática na Educação (CBIE 2016)

Anais do XXVII Simpósio Brasileiro de Informática na Educação (SBIE 2016)

analogias e personagens. A história apresentada no jogo é centrada em uma cidade, chamada Interacionópolis, onde vivem desenvolvedores e designers produzindo sistemas. Porém, surge uma bruxa, chamada Ruptura, que se incomoda com o sucesso dos desenvolvedores e começa a inserir várias rupturas de interação nas interfaces dos sistemas gerados dentro da cidade.
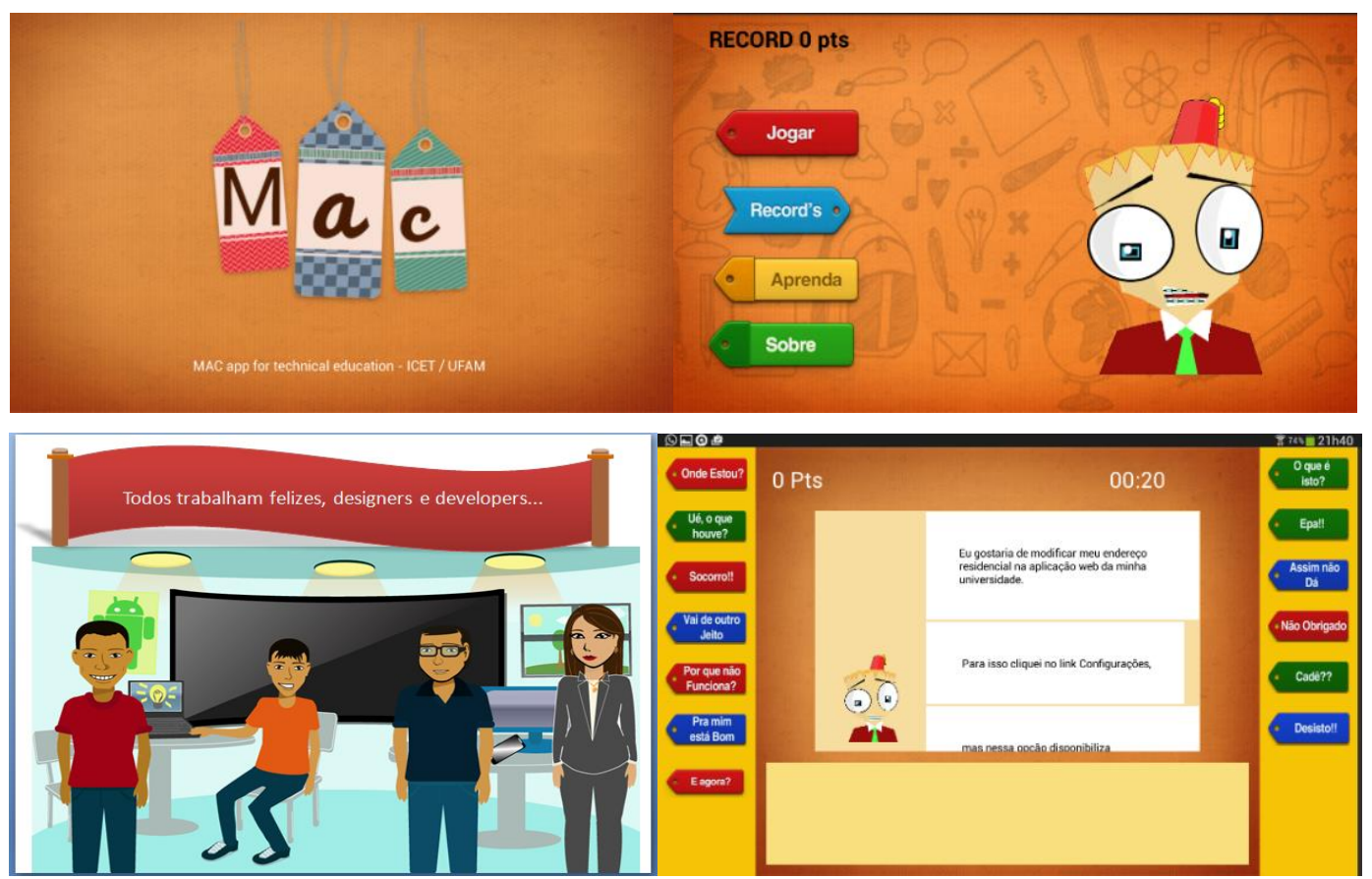

Figura 1. Interface Gráfica do Aplicativo MACteaching.

Para resolver a situação, uma equipe de desenvolvimento resolve aplicar o Método de Avaliação de Comunicabilidade (MAC). O jogo então tem o propósito de identificar as rupturas nos sistemas, onde o jogador do aplicativo atua como personagem principal, um participante chamado Meckinho. O objetivo é identificar, para cada ruptura qual a etiqueta do método que expressa o problema de comunicação na interface gráfica dos sistemas interativos.

Para a interação realizada pelo jogador durante a simulação, foi utilizado um mecanismo de drag-and-drop. O objetivo do jogo é conseguir etiquetar o máximo de rupturas corretamente e salvar a cidade da bruxa Ruptura. $\mathrm{O}$ jogo disponibiliza ainda descrições das treze etiquetas do MAC, com interface intuitiva e amigável. A versão inicial do jogo possui um nível iniciante e intermediário até o momento.

\section{Metodologia de Avaliação do Jogo}

Para entender a aplicabilidade do jogo MACteaching, como ferramenta capaz de estimular e contribuir com a construção de conhecimento e habilidades dos estudantes em relação ao MAC, foi realizado um estudo de observação. O propósito desse estudo foi avaliar, de forma qualitativa e quantitativa a contribuição do aplicativo no ensino do MAC. Para isso, foi realizado um estudo experimental com estudantes da disciplina de IHC, no Curso de Sistemas de Informação em uma universidade no norte do país. Este 
V Congresso Brasileiro de Informática na Educação (CBIE 2016)

Anais do XXVII Simpósio Brasileiro de Informática na Educação (SBIE 2016)

estudo focou em três aspectos de interação: a utilidade percebida, a facilidade de uso e a contribuição para o aprendizado, dentro da disciplina IHC, como ferramenta de apoio.

Além disso, o estudo serviu como base para avaliação das funcionalidades e da interface gráfica inicial do MACteaching. As sugestões dos participantes serviram de base para melhoria do aplicativo. Para a condução do estudo, foi elaborado um conjunto de etapas, apresentado na Tabela 1.

Tabela 1. Etapas do Processo de Avaliação do MACteaching.

\begin{tabular}{|c|c|c|}
\hline Etapas & Descrição & Atividades \\
\hline \multirow{4}{*}{ 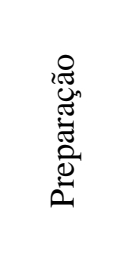 } & \multirow{4}{*}{$\begin{array}{l}\text { Etapa cujo objetivo foi elaborar o } \\
\text { cenário de avaliação e todos os } \\
\text { artefatos utilizados para a execução } \\
\text { do estudo. }\end{array}$} & P.1. Preparação do conteúdo \\
\hline & & P.2. Montagem do Ambiente de Avaliação \\
\hline & & P.3. Definição dos Participantes \\
\hline & & P.4. Construção dos formulários de avaliação \\
\hline \multirow{2}{*}{ 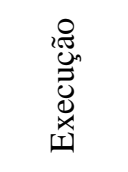 } & \multirow{2}{*}{$\begin{array}{l}\text { Realização do estudo, com atividade } \\
\text { em laboratório. Os alunos foram } \\
\text { monitorados sob supervisão do } \\
\text { professor da disciplina }\end{array}$} & $\begin{array}{l}\text { E.1. Realização da avaliação junto com os } \\
\text { participantes }\end{array}$ \\
\hline & & E.2. Observação pelo observador da avaliação \\
\hline \multirow{2}{*}{ 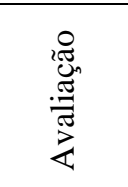 } & \multirow{2}{*}{$\begin{array}{l}\text { Coleta e análise dos aspectos } \\
\text { motivacionais ao longo do curso }\end{array}$} & $\begin{array}{l}\text { A.C.1 Coleta dos dados por meio de formulários de } \\
\text { avaliação de aprendizado e satisfação de uso. }\end{array}$ \\
\hline & & $\begin{array}{l}\text { A.C.2. Análise e avaliação da contribuição do } \\
\text { MACteaching para o aprendizado dos alunos. }\end{array}$ \\
\hline
\end{tabular}

Adicionalmente, foram elaborados os seguintes documentos e formulários: o Termo de Consentimento Livre e Esclarecido (TCLE) para garantir a segurança dos dados dos participantes; Formulário de Caracterização para identificar o grau de conhecimento e experiência dos participantes em IHC e no uso do MAC e; os Questionários e Formulários Pós-Avaliação para coletar a experiência de uso e percepção de aprendizado dos alunos após o teste. Como infraestrutura para realização do estudo foi escolhido um dos laboratórios de uma Instituição de Ensino Superior do norte do país.

\subsection{Execução da Avaliação}

O estudo foi realizado, no contexto da disciplina de IHC, teve a participação de um observador, responsável pelo desenvolvimento do jogo, responsáveis por observar as dificuldades de interação dos usuários com o aplicativo. E também o professor da disciplina, que foi responsável por supervisionar o estudo aplicado.

Para a avaliação inicial do jogo, apenas seis participantes se disponibilizaram voluntariamente em avaliar MACteaching. Apesar do número relativamente pequeno, estudos afirmam que é possível ter um resultado satisfatório na avaliação de uma tecnologia de software [Meireles e Bonifácio 2015], [Coutinho et al. 2014], [Prates e Barbosa 2007]. Após o uso do aplicativo, foram feitas entrevistas e aplicados questionários de avaliação sobre a experiência de uso e aprendizado dos participantes com o MACteaching. Os resultados foram agrupados em pontos fortes, pontos a serem melhorados e a percepção do participante ao usar o aplicativo como ferramenta de apoio ao ensino do Método de Avaliação de Comunicabilidade. 
V Congresso Brasileiro de Informática na Educação (CBIE 2016)

Anais do XXVII Simpósio Brasileiro de Informática na Educação (SBIE 2016)

\subsection{Resultados Obtidos}

Após a etapa de execução do estudo, foram coletadas as informações as quais geraram gráficos demonstrativos agrupando informações a respeito da facilidade de uso, aprendizagem do Método de Avaliação de Comunicabilidade e sugestões e melhorias para o MACteaching.

As escalas foram baseadas no Modelo de Aceitação de Tecnologia, para o uso de escalas de likert, que permitiram melhor análise quantitativa acerca dos resultados obtidos, conforme apresentado na Figura 2.

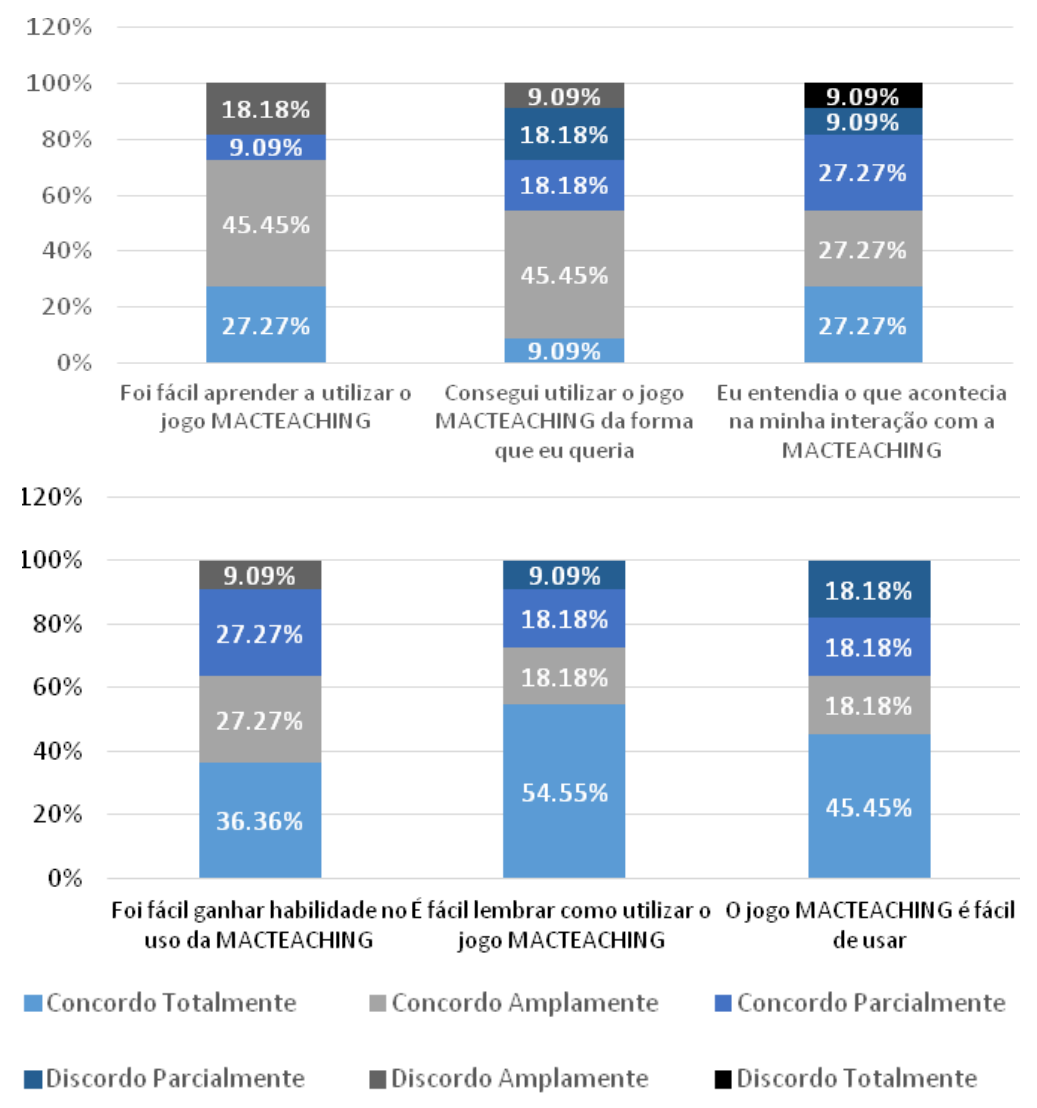

Figura 2. Gráfico de Satisfação de Uso do MACteaching.

De acordo com as informações obtidas, considerando a facilidade de uso, os participantes afirmaram conseguir utilizar o MACteaching de forma satisfatória. Cerca de 55\% dos participantes afirmaram ser fácil de lembrar como utilizar o jogo. Além disso, $36 \%$ do alunos relataram o ganho de habilidade no uso do MACteaching, e $45 \%$ que afirmaram que o MACteaching é fácil de utilizar. Os dados mostram indícios que a aceitação do aplicativo, considerando a interface gráfica e usabilidade. Em relação a aprendizagem do MAC, com o uso do aplicativo, os resultados mostraram importante correlação entre os conceitos aplicados no MACteaching e os do método original. A maioria os alunos afirmaram lembrar, compreender e entender sobre como aplicar na prática a etiquetagem do MAC após interação com o MACteaching. Cerca de 4 dos 6 afirmaram que a experiência de uso e aprendizado é mais intuitiva após o uso do aplicativo, conforme a Figura 3. 
V Congresso Brasileiro de Informática na Educação (CBIE 2016)

Anais do XXVII Simpósio Brasileiro de Informática na Educação (SBIE 2016)

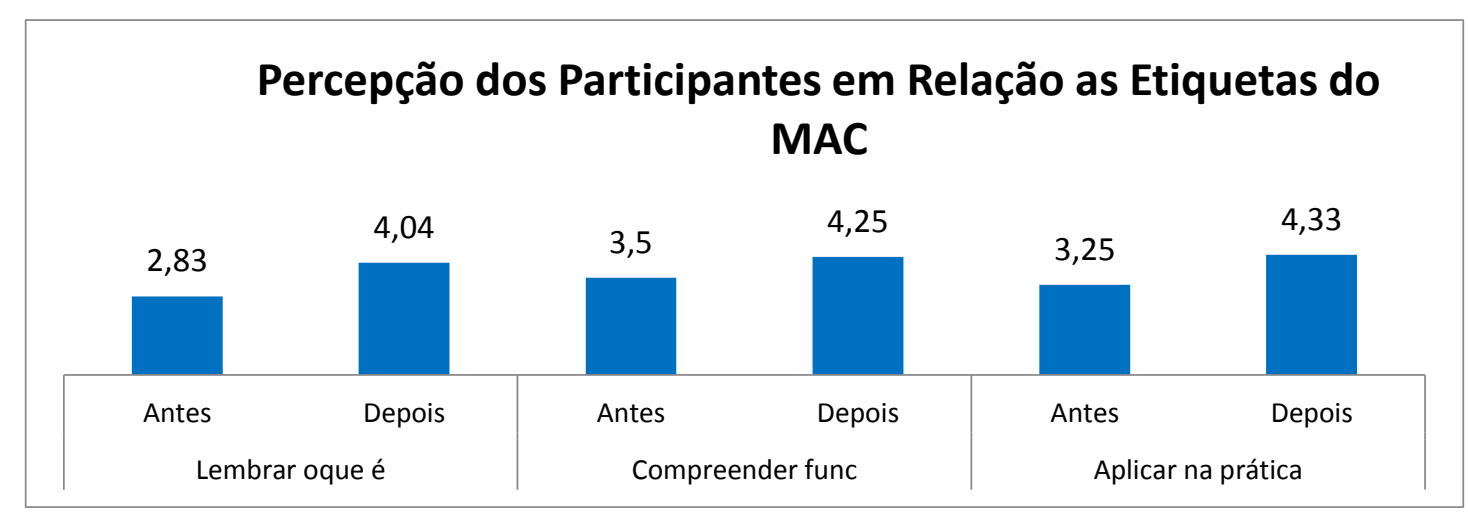

\section{Figura 3. Gráfico de Experiência de Aprendizado em relação ao} MACteaching.

O estudo também indicou os pontos fortes e de melhoria do aplicativo. Em relação aos pontos fortes, pode-se citar, o aluno 1 que afirmou: "A aplicação é uma ótima ferramenta para o aprendizado e assimilação da técnica MAC”, o aluno 4: "É iterativo, deixando o usuário com vontade de querer jogar" e o aluno 5: "Aprendi onde devo utilizar as etiquetas". Outro ponto observado, durante a avaliação, foi que a maioria dos alunos mencionaram a aprendizagem do MAC como ponto forte do jogo.

No aspecto de sugestões e melhorias, a maioria dos alunos se sentiram prejudicadas pela falta de feedback da aplicação em relação as suas respostas, no jogo. Podemos destacar, a afirmação do alunos 1 que relatou: "A aplicação deveria informar ao usuário uma mensagem informando que o mesmo selecionou a resposta incorreta", pelo aluno 2 "Falta de resposta ao erro", e do aluno 4, que relatou: "Não aparecem mensagens quando erramos". Além disso, foram observadas dificuldades dos participantes na interação com o aplicativo, principalmente para mover as etiquetas do método. Outro ponto mencionado foi a disputa com outros jogadores, que pode tornar o jogo mais atrativo.

Em relação a aprendizagem do MAC, utilizando o jogo as afirmativas foram favoráveis para o aplicativo. Segundo os resultados, todos participantes afirmaram lembrar, compreender e aplicar a etiquetagem de forma mais intuitiva após jogarem o MACteaching. Em relação ao conteúdo, os principais pontos destacados no estudo foi em relação a aprendizagem do MAC e a satisfação em jogar o MACteaching, conforme a Figura 4. 
V Congresso Brasileiro de Informática na Educação (CBIE 2016)

Anais do XXVII Simpósio Brasileiro de Informática na Educação (SBIE 2016)

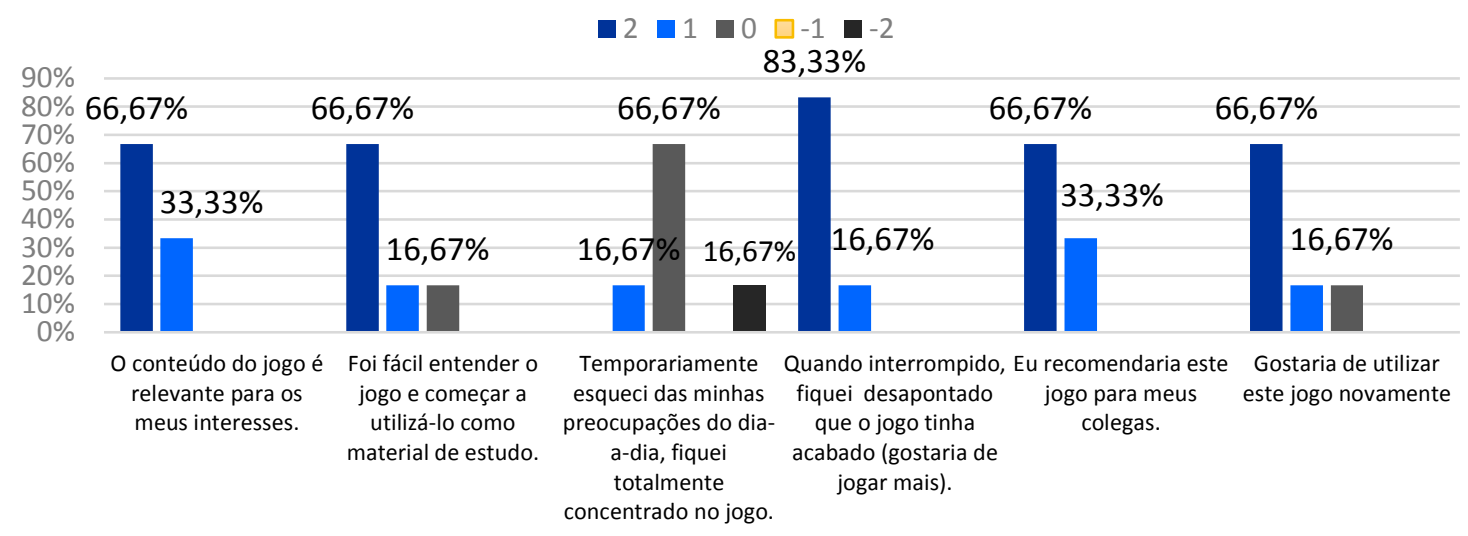

Figura 4 Gráfico de opinião sobre recomendações para o aplicativo.

Cerca de $70 \%$ dos alunos afirmaram que o aplicativo apresenta conteúdo de interesse e compreensão para aplicar o MAC. Por fim, os dados mostram que a maioria dos participantes do estudo afirmaram recomendar o MACteaching para outros estudantes. Essas informações serviram de base para realizar melhorias no aplicativo. Além de avaliar o resultado do MACteaching como ferramenta de ensino.

\section{Lições Aprendidas e Melhorias Realizadas}

Como o propósito do estudo foi avaliar a viabilidade do aplicativo como ferramenta de ensino em IHC e Engenharia de Software, a principal contribuição desse estudo inicial foi a identificação de pontos de melhoria na interface gráfica do aplicativo. Foram identificados um total de cinco pontos de melhorias como: dificuldade em saber para onde arrastar as etiquetas, a velocidade da história inicial do jogo, dificuldade de leitura em legendas do jogo; feedbacks do jogo sem destaque e; tempo de jogo muito curto. O estudo serviu de base para alteração na interface gráfica do aplicativo, como apresentado na Figura 5.
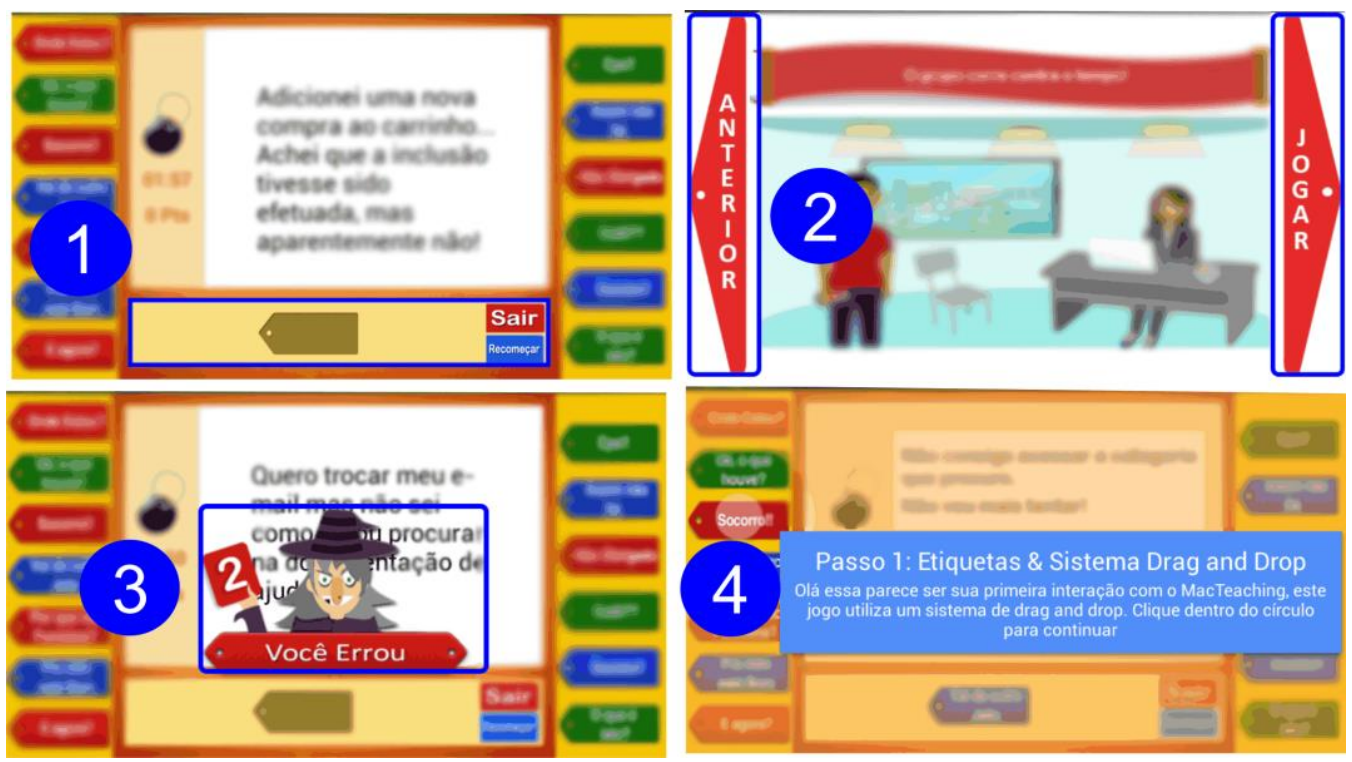

Figura 5. Alterações realizadas na interface gráfica do aplicativo após o estudo. 
A Figura 5 apresenta as melhorias realizadas no aplicativo tais como: indicação na interface gráfica do aplicativo mostrando o local de movimento das etiquetas (Figura 5-1); novos botões de controle avançar/jogar e voltar para melhor leitura da historinha do jogo (Figura 5-2); feedback após interação do jogo, com imagens que ilustram a resposta do usuário (Figura 5-3); aumento do tempo após iniciar a partida, além de ranking dos 10 (dez) melhores jogadores. E por fim, um tutorial sobre o funcionamento do jogo, mostrando instruções e pontos que auxiliam no aprendizado do jogo e do MAC (Figura 5-4). A nova versão desenvolvida do MACteaching, ainda em desenvolvimento, se encontra disponível na loja de aplicativos Play Store³.

\section{Considerações Finais e Trabalhos Futuros}

Com a crescente evolução do sistemas interativos e o surgimento de novas tecnologias de software, o ensino de IHC se torna cada vez mais importante. Esse cenário faz com que as instituições busquem alternativas inovadoras no processo de ensino, visando melhorar a integração dos envolvidos com atividades pedagógicas mais colaborativas para tornar o processo de aprendizagem mais dinâmico e prazeroso.

Diante desse contexto, o presente artigo apresenta a criação de um aplicativo, em formato de jogo para o ensino de um dos importantes métodos de IHC, fundamentados na Engenharia Semiótica, o Método de Avaliação de Comunicabilidade (MAC). Como avaliação inicial, apresentada nesse artigo, foi adotada uma avaliação experimental aplicado em ambiente de ensino, em uma disciplina de IHC, no curso de Sistemas de Informação em uma universidade do norte do Brasil.

O estudo foi de extrema importância, pois auxiliou na detecção de rupturas que permitiram o aprimoramento tanto da interface gráfica, como os fluxos de interação do usuário com o aplicativo. Outro ponto importante a ser destacado, está no fato da realização da avaliação na fase de desenvolvimento do aplicativo, o que permitiu a correção das interfaces gráficas e ainda a identificação de novas funcionalidades a serem desenvolvidas e adicionadas ao aplicativo MACteaching.

Os resultados quantitativos se mostraram úteis para a identificação de melhorias. Porém a realização de entrevistas sobre a experiência de uso e aprendizado em sala de aula tornou melhor a compreensão sobre como os usuários utilizam o MACteaching, ao presenciar eventuais dificuldades. Por essa razão, a análise qualitativa foi determinante para entender o comportamento e dificuldades dos participantes, além de apresentar características relacionadas ao aprendizado do MAC apresentando indícios do MACteaching como uma solução para contribuição para o processo de ensino e aprendizagem.

Como trabalho futuro pretende-se, ainda: (1) desenvolver novos requisitos e novos fluxos para melhoria do aplicativo, transformando o MACteaching em um plataforma colaborativa, para permitir que profissionais da educação utilizem o aplicativo dentro e fora da sala de aula, com a possibilidade de customização das questões utilizadas no jogo, inseridas pelos próprios pesquisadores e educadores da área de IHC e Engenharia de Software; (2) e realizar uma nova avaliação para identificar o impacto real do aplicativo no ensino de IHC, comparando com a metodologia de ensino tradicional e utilizando o aplicativo. Espera-se com este artigo, contribuir para o processo de ensino e

\footnotetext{
${ }^{3}$ https://play.google.com/store/apps/details?id=macapp.ufam.icet.com.mac
} 
V Congresso Brasileiro de Informática na Educação (CBIE 2016)

Anais do XXVII Simpósio Brasileiro de Informática na Educação (SBIE 2016)

aprendizagem da Engenharia de Software e de IHC, buscando proporcionar melhor qualificação dos profissionais por meio da teoria aliada a prática, utilizando tecnologias de software e assim proporcionando a criação de práticas pedagógicas inovadoras de ensino.

\section{Referências}

Bim, S.A. Prates, R.O. Silveira, M.S. and Winckler, M.(2011) "Ensino de IHCAtualizando as Discussões sobre a Experiência Brasileira". In: Anais do XIX Workshop sobre Educação em Computação (WEI). SBC, Porto Alegre.

Shimada, H. e Kitajima, M. (2006) "Why Do Illustrations Promote Text Comprehension? Motivation Effect and Elaboration Effect." In: 5th International Conference of the Cognitive Science. Vancouver, British Columbia, Canada.

Mitchell, Alice; Savill-Smith, C. (2004) "The use of computer and video games for learning: A review of the literature." In: Learning and Skills Development Agency (LSDA). pp 12- 25 San Francisco. 2015.

Cardoso, L. and Sieckenius, C. (2007). Commest - Uma ferramenta de apoio ao Método de Avaliação de Comunicabilidade. III Conferência Latino-Americana de Interação Humano-Computador. 2007.

Prates, R.O. and Barbosa, S.D.J.(2007) "Introdução à Teoria e Prática da Interação Humano- Computador fundamentada na Engenharia Semiótica". In T. Kowaltowski \& K. Breitman(orgs) Jornadas de atualização em Informática.

Savi, R. and Ribas, V. (2008)"Jogos Digitais Benefícios e Desafios. Centro Interdisciplinar de Novas Tecnologias na Educação.

Meireles, M.C. and Bonifácio, B. A. (2015) "Uso de Métodos Ágeis e Aprendizagem Baseada em Problema no Ensino de Engenharia de Software: Um Relato de Experiência." In: XXVI Simpósio Brasileiro de Informática na Educação, 2015, Maceió, 2015. pp. 180-189.

Coutinho, W. Couto, E. Biase, C. Fernandes, P. S. and Bonifacio, B. A. (2015) "Improving an Educational Mobile Application Through Usability Evluation. In: Proceedings of International Technology" In: Education and Development Conference, 2015. Madrid, Spain, v. 1. pp. 5812-5820.

de Souza, C. S. (2005) The Semiotic Engineering of Human-Computer Interaction. Cambridge, MA: The MIT Press.

Salgado, C.C.L., Bim, A.S., and Souza, S.C. ( 2006) “Comparação entre os Métodos de Avaliação de Base Cognitiva e Semiótica" Simpósio Brasileiro sobre Fatores Humanos em Sistemas Computacionais (IHC), pp. 158 - 167.

Barbosa, J.D.S. Silva, S.B. (2010). Interação Humano - Computador. 1ra ed. Elsevier, Rio de Janeiro, 2010, pp. 385.

Sommariva, L. Benitti, F. B.V. and Dalcin, F.S. (2011). "UsabilityGame: jogo simulador para apoio ao ensino de usabilidade". In: Proceedings of the 10th Brazilian Symposium on Human Factors in Computing Systems and the 5th Latin American Conference on Human-Computer Interaction. Brazilian Computer Society, 2011. pp. 61-65. 